Extending their Monte Carlo simulation to three dimensions, the researchers said it elucidates the process and the mechanism of the nanoporosity evolution in selective dissolution.

HAILONG HUANG

\section{Molecular Random-Access Memory Cell Demonstrated}

As a result of the common computer user's demands, both the software and hardware industries are struggling to push technological resources to the farthest limits possible. However, the traditional limits appear to be not that far away, so research focuses on faster and smaller microprocessors, faster and larger memory, and larger data-storage capabilities, for example. Researchers at Yale and Rice Universities have developed and demonstrated devices that exhibit electronically programmable and erasable memory bits compatible with conventional threshold levels, but at a molecular monolayer level. They reported their work in the May 28 issue of Applied Physics Letters.

Electronic memories that operate at the charge limit (single electron effects) have been previously demonstrated by other research teams, but without addressing yet the dimensional limit of a single molecule, which this team now believes is achievable. They have observed a charge storage in a self-assembled nanoscale molecular device that is operated as a random-access memory with practical thresholds and output under natural ambient operation, and with a bit retention time of $15 \mathrm{~min}$. They said that their device can potentially be scaled to the single-molecule level.

The molecular systems investigated were Au-(1)-Au (1: 2'-amino-4-ethynylphenyl-4'ethynylphenyl-5'-nitro-1-benzenethiolate); Au-(2)-Au (2: 4-ethynylphenyl-4'-ethynylphenyl-2'-nitro-1-benzenethiolate); Au-(3)$\mathrm{Au}$ (3: 2'-amino-4-ethynylphenyl-4'ethynylphenyl-1-benzenethiolate); and $\mathrm{Au}-$ (4)-Au (4: 4-ethynylphenyl-4'-ethynylphenyl-1-benzenethiolate). The first two combinations were observed to change conductivity state upon application of a voltage pulse, indicating the responsible molecular moiety. Although the size of the nanoscale structures built was determined by the limitations of the lithographic technique used for defining the contacts, there are no indications in the observed characteristics that limitations exist in scaling down to one the number of molecules in the active region of the device, assuming that an appropriate fabrication scheme can be identified.

Claudiu Muntele

\section{Use of Area Array Detector for X-Ray Fluorescence Holography Allows Simultaneous Recording of the Full Hologram without Sacrificing Angular Accuracy \\ Holography has traditionally, as in the} case of lasers, been limited by the radiation wavelength, the source size, and the detector resolving power. X-ray fluorescence holography (XFH) has recently been studied as a method for creating holograms of bulk structures with atomic-scale resolution. The primary hurdle to developing $\mathrm{XFH}$ into a practical investigative tool has been the difficulty associated with measuring the fluorescent radiation with an acceptable signal-to-noise ratio. While typically a solid-state detector with energy discrimination is employed, researchers at the Sincotrone Trieste in Italy have developed a simpler method whereby an absorption filter is used in front of an area detector without energy resolution. They have increased their photon count rate by more than an order of magnitude without sacrificing angular accuracy.

As reported in the May 7 issue of Applied Physics Letters, the researchers performed an internal source XFH experiment using a position sensitive detector (i.e., an image plate with pixel size $150 \mu \mathrm{m}^{2}$ $\times 150 \mu^{2}$ ) along with an absorption filter to record the full hologram of a $\mathrm{CoO}$ single crystal. They employed an $8.0-\mathrm{keV}$ monochromatic $\mathrm{x}$-ray beam collimated to a $0.3 \mathrm{~mm}^{2} \times 0.3 \mathrm{~mm}^{2}$ spot. A $50-\mu \mathrm{m}$-thick Fe absorber was placed between the sample and the detector in order to create an energy window. The fluorescence emitted by the Co atoms was incident on this Fe foil, causing the elastically scattered primary x-ray beam along with Co k $\alpha$ radiation to be absorbed, so that only $\mathrm{Co}$ $\mathrm{k} \beta$ photons along with some inelastically scattered photons reached the detector. An intensity pattern was recorded by measuring the fluorescent radiation, leaving the sample in different directions, and an image of the (001) plane of Co atoms was reconstructed.

"An important point," said researcher Andrea Lausi, "is that this setup does not require complex or dedicated instrumentation. Since both area detectors and foil filters are readily available at synchrotron facilities, this simplified setup should open up XFH experiments to a much wider community."

The scientists found that the photon flux incident on the image plate, or detector, was $\approx 5 \times 10^{8}$ photons/s which represents a gain of approximately three orders of magnitude over XFH experiments that employ solid-state detectors. The entire image was recorded on the position sensitive detector with an angular resolution better than $0.1^{\circ}$ $\times 0.1^{\circ}$. They found that the recorded image was undistorted even though the measurement was performed at only a single photon energy, and images of the third, fourth, and fifth Co neighbors were visible up to a distance of greater than $7 \AA$ from the emitter. Furthermore, the real-space image obtained from the experimental hologram was in good agreement with simulated data.

The researchers are excited by the ability to record x-ray holograms on an area detector, and Milos Kopecky said, "The combination of fast data collection times and high angular accuracy, which has not been demonstrated before, creates the possibility of time-resolved holographic imaging. This is a promising tool for imaging the structures of small clusters or molecules."

STEFFEN K. KALDOR

\section{Dicyclopentadiene Serves as Automated Healing Agent of Cracks in Thermosetting Materials}

When thermosetting polymeric materials are damaged by mechanical loading or thermal fatigue, cracks develop which may continue to grow and compromise their structural integrity. Recently, a team of researchers from the University of Illinois at Urbana-Champaign have developed a material system with the ability to autonomically heal cracks. As reported in the February 15 issue of Nature, the system incorporates dicyclopentadiene (DCPD) as a healing agent into an epoxy matrix. The healing agent is microencapsulated within a urea-formaldehyde shell. When the cracks in the thermosetting material reach the 50-200 $\mu \mathrm{m}$ microcapsules, they rupture and release the healing agent into the crack plane. Once in the crack plane, the healing agent contacts an embedded catalyst, triggering polymerization of the healing agent and bonding the crack faces.

A transition-metal catalyst (Grubbs' catalyst) is used to initiate the living ringopening metathesis polymerization (ROMP) with the DCPD. The ROMP reaction satisfies the diverse requirements of the self-healing system such as long shelf life, low monomer viscosity and volatility, rapid polymerization at ambient conditions, and low shrinkage upon polymerization.

The researchers confirmed their work with a series of materials characterizations and analysis. The results of solution-state and solid-state nuclear magnetic resonance offered information on the stability and activity of the Grubbs' catalyst in the epoxy 